\title{
Effect of post curing, fibre content and resin-hardener mixing ratio on the properties of kenaf-aramid hybrid composites
}

\begin{abstract}
Polymer composites reinforced with high strength synthetic fibres have been used for many engineering applications. Environmental and economic issues, encourage the exploration on the introduction natural-synthetic fibre hybrid composites. Mechanical properties are critical to composite performance and may due to the manufacturing process conditions. This study investigates the effect of post curing temperature, natural fibre content and resin-hardener mixing ratio on mechanical properties of kenaf-Kevlar hybrid composites. A full factorial design (23) was carried out to determine the effect these factors on the responses: flexural strength, flexural modulus and impact strength. A statistical study has been performed in order to determine the how the factors affect the responses. The study showed that postcuring temperature, kenaf content and resin-hardener mixing ratio gives significant effects on the mechanical properties of kenaf-Kevlar hybrid composites.
\end{abstract}

Keyword: Design of experiment; Flexural modulus; Flexural strength; Hybrid composites; Impact strength 